\title{
1 Targeted next-generation sequencing of Candidate 2 Regions Identified by GWAS Revealed SNPs 3 Associated with IBD in GSDs
}

4 Atiyeh Peiravan ${ }^{1 *}$, Mazdak Salavati ${ }^{2}$, Androniki Psifidi $^{3}$, Mellora Sharman ${ }^{4}$, Andrew

5 Kent $^{5}$, Penny Watson ${ }^{5}$, Karin Allenspach ${ }^{6}$, Dirk Werling ${ }^{1 *}$.

$6{ }^{1}$ Department of Pathology and Pathogen Biology, Royal Veterinary College,

7 University of London, North Mymms, UK

$8{ }^{2}$ The Roslin Institute and Royal (Dick) School of Veterinary Studies, The University 9 of Edinburgh, Edinburgh, UK

$10{ }^{3}$ Department of Clinical Sciences and Servises, Royal Veterinary College, University

11 of London, North Mymms, UK

$12{ }^{4}$ VetCT, St John's Innovation Park, Cambridge, UK

$13{ }^{5}$ Queen's Veterinary School Hospital, University of Cambridge, Madingley Road, 14 Cambridge. CB3 0ES

$15{ }^{6}$ College of Veterinary Medicine, Iowa State University, Ames, USA

16 A. Kent's current address is Willows Referral Service, Highlands Road, Shirley,

17 Solihull, B90 4NH.

$18 *$ Corresponding authors

19 Email: apeiravan@rvc.ac.uk and dwerling@rvc.ac.uk

21 Keywords: Inflammatory bowel disease, Chronic enteropathy, NGS, GSD, Canine 


\section{Abstract}

24 Canine Inflammatory bowel disease (IBD) is a chronic multifactorial disease, resulting

25 from complex interactions between the intestinal immune system, microbiota and

26 environmental factors in genetically predisposed dogs. Previously, we identified

27 several single nucleotide polymorphisms (SNP) and regions on chromosomes (Chr) 7,

289,11 and 13 associated with IBD in German shepherd dogs (GSD) using GWAS and

29 FST association analyses. Here, building on our previous results, we performed a

30 targeted next-generation sequencing (NGS) of a two Mb region on Chr 9 and 11 that

31 included 14 of the newly identified candidate genes, in order to identify potential

32 functional SNPs that could explain these association signals. Furthermore, correlations

33 between genotype and treatment response were estimated. Results revealed several

34 SNPs in the genes for canine EEF1A1, MDH2, IL3, ILA, IL13 and PDLIM, which,

35 based on the known function of their corresponding proteins, further our insight into

36 the pathogenesis of IBD in dogs. In addition, several pathways involved in innate and

37 adaptive immunity and inflammatory responses (i.e. T helper cell differentiation, Th1

38 and Th2 activation pathway, communication between innate and adaptive immune

39 cells and differential regulation of cytokine production in intestinal epithelial cells by

40 IL-17A and IL-17F), were constructed involving the gene products in the candidate

41 regions for IBD susceptibility. Interestingly, some of the identified SNPs were present

42 in only one outcome group, suggesting that different genetic factors are involved in

43 the pathogenesis of IBD in different treatment response groups. This also highlights

44 potential genetic markers to predict the response in dogs treated for IBD. 


\section{Introduction}

46 Inflammatory bowel disease (IBD) is a common cause of chronic gastrointestinal

47 disease in humans and dogs. IBD is characterised by persistent or recurrent

48 gastrointestinal signs (GI) including chronic diarrhoea, vomiting and weight loss and

49 with histological evidence of inflammation in the lamina propria of the small intestine,

50 large intestine or both (Albert 1999). The diagnosis of IBD in both humans and dogs

51 is by exclusion, as several diseases can cause chronic gastrointestinal inflammation

52 secondarily (Guilford 1996; Hendrickson et al., 2002).

53 The pathogenesis of IBD is believed to be multifactorial in both humans and dogs,

54 caused by a complex interaction between the intestinal innate and adaptive immune

55 system, the intestinal microbiome, and the genetic make-up of an individual. Although

56 IBD can affect multiple dog breeds, breed-specific disease phenotypes and

57 associations have been reported (Kimmel et al., 2000; Simpson et al. 2006). In the

58 United Kingdom (UK), German shepherd dogs (GSD) have been reported to be at

59 increased risk of developing the disease (Kathrani et al., 2011).

60 IBD is not a curable disease, therefore the aim of current treatment approches is to

61 minimise the severity and frequency of the clinical signs. In general, treatment

62 protocols include dietary modifications (Luckschander et al. 2006; Mandigers et al.

63 2010), antibiotics (German et al., 2003; Westermarck et al., 2005), and corticosteroid

64 (Allenspach et al., 2007) treatment trials. As such, canine IBD is generally classified

65 only retrospectively based on response to treatment into food responsive

66 disease/diarrhoea (FRD), antibiotic responsive disease/diarrhoea (ARD), or steroid

67 responsive disease/diarrhoea (SRD), which are usually used interchangeably with

68 idiopathic IBD. Canine IBD patients are considered as FRD and ARD if their clinical 
69 signs improve or resolve following dietary modification and/ or antibiotic treatment.

70 Those that fail to respond to a change of diet and/ or antibiotic therapy require

71 immunosuppressive treatment (usually corticosteroids) to treat their clinical signs

72 (SRD) (Allenspach et al., 2007). To date, treatment with anti-inflammatory/

73 immunomodulatory medication such as corticosteroids is the mainstay treatment for

74 both human and canine IBD patients (German et al., 2003). However, up to $50 \%$ of

75 dogs with IBD that are initially managed with steroids will develop resistance and/or

76 significant side effects, which ultimately leads to euthanasia for many of them

77 (Allenspach et al., 2006, 2007).

78 Recent advances in clinical genetics make it possible to use the patients' genetic

79 profile to predict response to treatment (William and Sandborn 2004; Roberts and

80 Barclay 2012). Similar to human IBD, it is hoped that identifiyng the genes involved

81 in canine IBD will provide insights into disease pathogenesis in canine IBD. This

82 could lead to the development of genetic screening panels useful for both diagnosis

83 and identifying dogs that are more likely to fall into specific groups of treatments.

84 So far, studies of canine IBD using a candidate gene approach, have identified a

85 number of single nucleotide polymorphisms (SNP) associated with the disease in

86 genes encoding pattern recognision receptors (PRR) of the innate immunity ( Kathrani

87 et al., 2010, 2011, 2014) as well as associations between SNPs in Major

88 histocompatibility class (MHC) II haplotypes and a potentially increased resistance to

89 IBD in GSD (Peiravan et al., 2016). The release of the re-assembled dog genome and

90 development of high-density canine DNA SNP arrays have enabled several successful

91 GWAS studies aimed at investigating the genetic architecture of both monogenic and

92 polygenic complex diseases of the dog (Wood et al., 2009; Wilbe et al., 2010; Lequarré

93 et al., 2011; Tengvall et al., 2013). Previously, based on GWAS and Fst association 
94 analyses of IBD cases and controls, we identified several SNPs and regions on

95 chromosomes (Chr) 7,9,11 and 13 associated with IBD in GSD, including a total of

9680 genes. Using a combination of pathways analysis and a list of genes that have been

97 reported to be involved in human IBD, we identified 16 candidate genes potentially

98 associated with IBD in GSD (Peiravan et al. 2018).

99 Genome-wide association studies rely on the principal of linkage disequilibrium (LD).

100 While the extensive LD and long haplotype blocks (0.5-1.0 Mb) within breeds,

101 resulted from genetic bottlenecks during domestication of dogs and breed formation,

102 is an advantage in the initial GWAS it complicates the subsequent identification of the

103 causative variant(s) (Sutter 2004; Karlsson et al., 2007). The association signals

104 identified through GWAS represent most likely markers that are not the causal variants

105 themselves but are linked instead with nearby causative polymorphisms. Therefore, in

106 order to generate a hypothesis about mechanisms underlying a specific phenotype, it

107 is important to detect the causal variants themselves.

108 In the present study, we performed a targeted NGS of $2 \mathrm{Mb}$ regions on Chr 9 and 11,

109 which include 14 of the newly identified candidate genes, aiming to identify potential

110 functional SNPs that could explain the GWAS association signal. We also investigated

111 whether there was a correlation between the identified SNPs and response to treatment

112 in the IBD cases, used in this study. 


\section{Materials and methods}

\section{Ethics and welfare statement}

115 All blood samples used in this study were collected in ethylenediaminetetraacetic acid

116 (EDTA) and represented residual material following completion of clinical diagnostic

117 testing. Residual samples were utilised for research with informed owner consent.

118 The use of these residual EDTA blood samples and buccal swab samples within the

119 current study was approved by the RVC Animal Welfare Ethical Review Body

120 (AWERB; approval number 2013 1210).

\section{Selection of cases and controls for targeted sequencing}

122 IBD cases and controls were identified based on inclusion criteria described

123 previously (Peiravan et al., 2018). A follow-up on all cases and controls was

124 performed by contacting the referring veterinary surgeons and/or owners to gather

125 information on treatment response of the dogs, including assessment of any changes

126 to the course of treatment, and if so what the response to the new treatment was.

127 A total number of 48 GSDs with adequate followup information and available 128 genomic (g)DNA samples were enrolled, including 28 cases (diagnosed with IBD) 129 and 20 controls (non-inflammatory disease). Among the IBD cases, there were 9 FRD, 1304 ARD, 11 SRD and 4 NRS/PTS (No Response to Steroid, PTS: Put To Sleep) cases.

131 Control dogs were breed-matched that were presented with variety of non132 inflammatory conditions or no-known diseases.

\section{SureSelect XT Library preparation and sequence capture}

134 A custom-designed sequence capture array (SelectSure XT custom 0.5-2.9 Mb, 135 Agilent), was designed and manufactured by Agilent, in order to isolate the targeted 
136 region identified on $\mathrm{Chr} 9$ and $11,1 \mathrm{Mb}$ up- and downstream of the most significant

137 SNPs identified by the previous GWAS (Peiravan et al., 2018), from total gDNA,

138 using start and end coordinates of the associated regions. Properties of the final design

139 of each array designed for the capture of the target regions on Chr 9 and 11 are shown

140 in Table 1.

141 For each gDNA sample to be sequenced, an individual indexed library was prepared.

142 DNA libraries were prepared using the SureSelect XT Library Prep Kit (Agilent)

143 following the manufacturer instructions. In brief, $200 \mathrm{ng}$ of the gDNA samples were

144 fragmented by sonication and the paired-end adaptors were ligated to the blunt-ended

145 fragments using the SureSelect XT Library Prep Kit ILM. Then, the adaptor-ligated

146 fragments were PCR amplified, purified and hybridyzed to the capture array, using the

147 SureSelectXT DNA kit following, the manufacturer instructions. Then, unbound

148 fragments were washed away. Subsequently, the SureSelect- captured DNA libraries

149 were eluted, purified and PCR amplified using an individual indexing primer for each

150 sample. Following quality control, captured libraries were sequenced with a read

151 length of $150 \mathrm{bp}$ (paired-end reads) on an Illumina NextSeq (500 Mid Output)

152 platform. Sequencing was performed at the Genomic Centre of Queen Mary

153 University of London (QMUL).

\section{Analysis of sequencing data}

155 The Genome Analysis Toolkit (GATK 3.8) (https://software.broadinstitute.org/gatk)

156 best practice workflow, in house bash and R scripting was used for processing of raw

157 sequencing data. BAMStats 2.1, which provides descriptive statistics for various

158 metrics, was used to calculate average coverage. 
159 Raw sequencing data were visualised and inspected using FastQC (v0.11.6)

160 (http://www.bioinformatics.babraham.ac.uk/projects/fastqc/). Thereafter, reads were

161 trimmed and filtered using Trimmomatic (v0.36) (Bolger et al., 2014). The trimmed

162 reads were aligned and mapped to the canine genome (CanFam3.1), using Burrow-

163 wheeler aligner (BWA, v0.7.15) with default parameters (bwa mem -M -t -R). BWA-

164 MEM is designed for aligning relatively short sequence reads ranging from 70 bp to 1

$165 \mathrm{Mb}$ against a long reference and is generally recommended for high-quality queries

166 (Li and Durbin 2009). Once the reads were mapped to the canine genome and merged,

167 they were sorted by the coordinates using the "samtools sort" command (Li and Durbin

168 2009).

169 Potencial PCR duplicates were flagged in the read's SAM record using Picard tool

170 (hosted by SAMtools), so that duplicates could be identified during downstream

171 processing. Most GATK tools will then ignore the flagged reads by default, through

172 the internal application of a read filter. Base quality score was adjusted according to a

173 model of covariations bulit based on the data and a set of known variants

174 (https://m.ensembl.org/info/genome/variation/species/sources_documentation.html\#c

175 anis_lupus_familiaris).

176 Genetic variants were called individually on each sample's BAM file(s) using the

177 HaplotypeCaller (GATK 3.8) in -ERC gVCF mode to produce an intermediate file

178 format called gVCF file (genomic VCF). Following variant calling, the GVCFs of

179 multiple samples were run through joint genotyping to produce a multi-sample VCF

180 callset, using GenotypeGVCFs. Then, the raw SNPs extracted from the multi-sample

181 VCF callset produced at genotyping stage, was subject to hard filtering. All the scripts

182 that were used for variant calling, genotyping, and filtering annotations and values 
183 recommended by GATK best practice for hard filtering are shown in Supplementary

184 Table A1.

\section{Analysis of variants}

186 The coordinate positions of the filtered SNPs were used to categorise them into

187 different groups; a) SNPs only present in cases, b) SNPs only present in controls and

188 c) SNPs present in both cases and controls. The latest group was further divided into

189 SNPs with the same alternate allele in both populations and those with different

190 alternate in case or control population.

191 SNPs in each group were then annotated using variant effect predictor (VEP,

192 https://www.ensembl.org/Tools/VEP). The effect of SNPs on genes, transcripts, and

193 protein sequence, as well as regulatory regions were determined and SIFT predictions

194 of the effects of SNPs (tolerated or deleterious) were also acquired through VEP. The

195 focus was on detection of exonic SNPs with high importance such as non-sense (stop-

196 gain) and missense (non-synonymous) exonic and splicing, since these can affect

197 considerably the function of the encoded protein. In addition, genomic regions located

198 within $1 \mathrm{~Kb}$ up- and downstream of the candidate genes were analysed in order to

199 detect SNPs with putative regulatory effect. For the case-control (overlapped) group

200 with the same alternate allele in case and control group, high impact SNPs with

201 deleterious SIFT score were detected and then the frequency of the identified SNPs

202 copmpared between the two groups to identify those with statisticaly significant

203 difference between cases and controls. The integrative genomic viewer (IGV 2.4.) was

204 also used for manual visualisation of SNPs. 


\section{Statistical analysis}

206 Allele frequencies were calculated and compared using VassarStats (Web Site for

207 Statistical Computation, Vassar College, Poughkeepsie, NY,

208 http://www.vassarstats.net/odds2x2.html). Two-way contingency tables were used to

209 calculate two-tailed Fisher's exact probability statistic for association of each allele

210 with disease status. Statistical significance was set at a $\mathrm{p}$ value of $<0.05$. The

211 calculation of Hardy-Weinberg equilibrium (HWE) for the identified variants was

212 carried out performing chi-square test using the SNPSTATS programme

213 (http://bioinfo.iconcologia.net/SNPstats).

\section{Pathway and enrichment analysis}

215 The candidate gene lists for IBD susceptibility were analysed using the IPA

216 programme (www.ingenuity.com) in order to identify canonical pathways and gene

217 networks constructed by the products of the genes. IPA builds several possible

218 pathways and networks serving as hypotheses for the biological mechanism

219 underlying the data based on a large-scale causal network obtained from the Ingenuity

220 Knowledge Base, which are subsequently summarised by the identification of the most

221 suitable pathways and networks based on their statistical significance.

222 In addition to IPA analysis, a second approach was used to identify the best candidate

223 genes associated with IBD in GSDs, using Enrichr, which is a web-based enrichment

224 analysis tool (http://amp.pharm.mssm.edu/Enrichr). For this analysis, the default

225 statistical tests and corrections for multiple testing to maintain an overall $\mathrm{p}$ value of

2260.05 were applied. 


\section{Association of variants with treatment response}

228 To investigate whether there was a correlation between variants identified and

229 response to treatment, the 28 IBD cases that were used in the current study were

230 categorised into two groups as shown in Table 2.

\section{Results}

\section{Quality control of gDNA, captured DNA libraries and sequencing data}

233 The results of Qubit system, used to quantify gDNA before library preparation, are

234 shown in Supplementary Table A2. The quality and size of fragmented DNA was

235 assessed with D1000 ScreenTape on Agilent 2200 TapeStation. The electropherogram

236 produced for each sample confirmed that the DNA samples had been fragmented to

237 the required size with the shearing size range of 100 to $500 \mathrm{bp}$, with a peak at 150-200

238 bp. The concentration of the amplified DNA libraries after capture, the number of

239 libraries and their size are shown in Supplementary Table A3. The electropherogram

240 produced for each sample showed a distribution with a DNA fragment size peak of 241 approximately 225 to $275 \mathrm{bp}$.

242 All samples were successfully sequenced and passed internal QC at the genomic centre

243 of QMUL. The distribution of coverage was similar between the two target regions.

244 Average mapped-sequencing coverage was $256 \times$ and $230 \times$ for $97.5 \%$ of the regions

245 on Chr 9 and 11 respectively (Supplementary Table B). There was no significant

246 differences in coverage rates between cases and controls on any of the two target

247 regions. Average coverage of the target region on Chr 9 was $254 \times$ and $258 \times$ for cases

248 and controls respectively. For the target region on Chr 11, the average coverage was

$249228 \times$ for cases and $233 \times$ for controls. 


\section{Analysis of variants and variant annotation}

251 The number of raw SNPs within the target regions on Chr 9 and 11 and the number of

252 SNPs that passed hard filtering are shown in Error! Reference source not found. .

253 Error! Reference source not found. shows the number of SNPs in each group per

254 chromosome. SNPS identified in each group and their effect and properties are

255 presented in the Supplementay Tables C and D.

\section{Variants on Chromosome 9}

257 Twelve variants with high or moderate impact, which were deleterious based on their

258 SIFT score, were detected only in cases (Error! Reference source not found.Error!

259 Reference source not found.). Among these, there were two stop_ gained variants

260 with high impact. The remaining ten variants were missense variants with moderate

261 impact. In addition, 48 variants with modifier impact were identified within $1 \mathrm{~kb}$ up-

262 and downstream of the candidate genes present only in cases (Supplementary Table

263 C).

264 Thirty missense variants (2 known and 28 new) with moderate impact that were

265 deleterious according to their SIFT score were detected only in controls. In addition,

26665 variants with modifier impact (13 known and 52 new) were observed within $1 \mathrm{~kb}$

267 up- and downstream of genes (Supplementary Table C).

268 Twenty-six missense variants with moderate impact were identified (14 known and 12

269 new) in both cases and controls. However, no significant differences in the frequency

270 of these variants was detected between cases and controls (Supplementary Table C).

$271 \quad$ Variants on Chromosome 11

272 One new missense SNP (deleterious based on SIFT score, SIFT: 0.03) was found only

273 in cases, and this SNP seems to have a moderate impact in a novel protein coding gene 
274 that is orthologous to human ATP5MF (ATP synthase membrane subunit F; for more

275 details, please see Supplementary Table D). In addition, five SNPs (3 known and 2

276 new) were identified within $1 \mathrm{~kb}$ upstream of gene TSS coordinates. In addition, 12

277 SNPs (3 known and 8 new) were detected within $1 \mathrm{~Kb}$ downstream of the hypothesised

278 candidate genes. Interestingly, associations between several of these candidate genes

279 and human IBD have already been described. The details of the identified variants are

280 shown in Supplementary Table D.

281 Two of these SNPs with modifier impact were located within $1 \mathrm{~kb}$ upstream of IL3, a

282 haemopoietic cytokine driving the development of myeloid stem cells that was

283 previously identified to be associated with IBD (Peiravan et al. 2018). Two SNPs were

284 found downstream of PDLIM (a protein with cysteine-rich double zinc fingers

285 involved in protein-protein interaction and cytoskeletal organisation) and IL13 (a Th2

286 cytokine involved in IgE synthesis, chitinase upregulation and hyperresponsiveness of

287 mucosal surfaces) and one new SNP was found downstream of IL4 (a Th2 cytokine

288 produced by mast cells, eosinophils and basophils that stimulates B cells into plasma

289 cells and shares functions similar to IL-13). All of these genes have already been

290 reported to be associated with human IBD in previous studies. SNPs with a statistically

291 significant difference ( $\mathrm{p}$ value $<0.05$ ) between cases and controls are shown in Error!

292 Reference source not found.6.

293 Two new missense SNPs, deleterious based on SIFT score, were identified in a protein

294 coding gene, orthologous to human HINT1 (histidine triad nucleotide binding protein

295 1) in the control population. HINT1 is a hydrolase and gene ontology annotations

296 related to this gene include nucleotide binding and protein kinase $\mathrm{C}$ binding.

297 Furthermore, five SNPs (1 known and 4 new) within $1 \mathrm{~Kb}$ upstream and ten SNPs (1

298 known and 9 new) within $1 \mathrm{~Kb}$ downstream of the candidate genes and Small nucleolar 
299 RNAs (snoRNA) on Chr 11 were identified in the control population. Two SNPs

300 downstream of a snoRNA were observed to have significant P value (Supplementary

301 Table D).

302 Ten missense SNPs with high and moderate impact were detected in both cases and

303 controls. However, only one SNP in IL13 was found to be deleterious based on the

304 SFIT score and there was no statistically significant difference in the frequency of this

305 SNP between case (Maf:0.054) and control group (Maf:0.074)(Error! Reference

306 source not found.7).

307 Pathway and Network analysis reveal impact on genes involved in

308 inflammatory response and metabolism

$309 \quad$ IPA analysis

310 Several pathways involved in innate and adaptive immune and inflammatory response

311 (i.e. T helper cell differentiation, Th1 and Th2 activation pathway, communication

312 between innate and adaptive immune cells and differential regulation of cytokine

313 production in intestinal epithelial cells by IL-17A and IL-17F) and metabolism (i.e.

314 TCA cycle II (Eukaryotic), gluconeogenesis I, aspartate degradation II and pyrimidine

315 deoxyribonucleotides De Novo biosynthesis) were constructed by the gene products

316 in the candidate regions for IBD susceptibility (Error! Reference source not found.

317 and Supplementary Table E ). Moreover, four networks of molecular interactions

318 related to cell cycle (IPA Score: 22) (Error! Reference source not found.), hereditary

319 disorder and metabolic disease (IPA Score: 17), cellular movement (IPA Score: 14)

320 and small molecule biochemistry and metabolism (IPA Score: 12) were constructed,

321 using the list of candidate genes, located in the targeted regions for IBD

322 (Supplementary Table E). 


\section{Enrichment pathway analysis}

324 The results of the Enrichr analysis showed that several genes within our list appear to

325 be involved in biological processes and/or are molecular components that have been

326 associated or directly/indirectly involved in human IBD. Results of the pathway

327 association analysis using different databases and details of pathways and genes

328 involved in each pathway are shown in Supplementary Table F.

\section{Association of variants with treatment response}

330 The majority of SNPs identified in the candidate regions for IBD were present in FRD,

331 ARD and the steroid treated group, including SRD and those dogs that were

332 euthanized (NRS/PTS). Several missense and modifier SNPs were present in either

333 FRD and ARD (20 SNPs) or the steroid treated group (10 SNPs) including SRD and

334 NRS/PTS dogs, however, these SNPs were present in only one or two IBD cases (more

335 details are shown in Supplementary Table F).

\section{Discussion}

337 In the present study, we performed a targeted NGS experiment in previously identified

338 candidate region for canine IBD susceptibility, in order to detect good candidate genes

339 and mutations. The vast majority of variants identified were novel variants. Some of

340 the SNPs identified were not in HWE. Deviation from HWE in case-control genetic

341 association studies is indicative of genetic association (Ziegler et al., 2011). However,

342 intensive selective breeding during breed formation, and therefore loss of random

343 mating that would normally enrich gene pool and maintain HWE, may also explain

344 why some of the SNPs were not in HWE (Ziegler et al., 2011). In addition, due to the

345 small sample size used in this study and missing calls in some of the SNPs that could

346 affect the significance of each genotype, some SNPs may not be in HWE. It is worth 
347 mentioning that a number of SNPs identified in the case population were only seen in

348 the heterozygote state, such as those in EEF1A1 (Elongation Factor 1 Alpha). One

349 possible explanation could be that homozygote SNPs might have been present but

350 have not been captured (low quality/ filtered out etc). Another possibility is that the

351 heterozygotes were introduced recently by either random mutation or outbreeding of

352 GSDs, as heterozygosity in each breed is very low (Lindblad-Toh et al., 2005). It may

353 also be possible that a homozygote state could be lethal.

354 Two novel stop-gained SNPs in EEF1A, were identified only in cases and were present

355 in 26 out of 28 cases. EEF1A1 is an important protein that initiates protein translation

356 elongation and triggers the initiation of protein translation elongation (Kapp and

357 Lorsch 2004; Schulz et al., 2014). Apart from its canonical function in translation

358 elongation by ribosomes, EEF1A1 plays an important role in a wide variety of cellular

359 processes including signaling transduction, heat shock response, cytoskeleton

360 regulation (Negrutskii et al., 2012) and cellular apoptosis (Kobayashi and Yonehara

361 2009). It has been also documented that binding of EEF1A1 to STAT3 is crucial for

362 STAT3 phosphorylation and for NF- $\kappa$ B/STAT3 activation, which enhances IL-6

363 expression (Schulz et al., 2014). Elevated levels of this cytokine were reported to play

364 a pivotal role in the initiation of inflammatory processes and progression of disease in

365 many clinical conditions including rheumatoid arthritis, Alzheimer's disease and

366 Crohn's disease (Ito 2004; Cacquevel et al., 2004; Nishimoto 2006; Murphy et al.,

367 2012). However, no previous association with IBD has been reported.

368 MDH2 (Mitochondrial malate dehydrogenase) is another good candidate gene located

369 on Chr 9, since several missense mutations were detected in this gene, and were only

370 present in IBD cases. In addition, IPA analysis showed that this gene was part of a 371 gene network involved in hereditary disorders and metabolic diseases, as well as 
372 involvement in pathways associated with metabolism. The mitochondrial malate

373 dehydrogenase, encoded by $M D H 2$, is a mitochondrial protein, which plays an

374 important role in energy production. Altered expression of MDH2 has been reported

375 in studies investigating differentially expressed proteins in intestinal biopsies of IBD

376 patients. Down-regulation of mitochondrial proteins involved in energy production

377 including MDH2 in the colonic mucosal biopsies of ulcerative colitis (UC) patients

378 was previously reported by Hsieh et al. (Hsieh et al., 2006). Results of this study

379 suggested the implications of colonocyte mitochondrial dysfunction and perturbed

380 mucosa immune regulation in the pathogenesis of UC.

381 In the control population, two known SNPs (rs850782880 and rs852254668) that were

382 identified within less than 300bp downstream of the MRPS2 gene, were of particular

383 interest. These two SNPs were present in all 20 control GSDs used in this study,

384 therefore could potentially be considered as protective variants for IBD in GSDs.

385 MRPS2 encodes mitochondrial ribosomal protein subunit 2, which is involved in

386 protein synthesis within the mitochondrion. Most of the mitochondrial proteins

387 including the ribosomal proteins and translation factors that are responsible for the

388 expression of the mitochondrial genome, are synthesized on cytoplasmic ribosomes

389 and imported into mitochondria post-translationally. The mitochondrial oxidative

390 phosphorylation system, which produces the bulk of ATP for almost all eukaryotic

391 cells to sustain cells' normal functions, depends on the translation of 13 mitochondrial

392 DNA (mtDNA)-encoded polypeptides by mitochondria-specific ribosomes in the

393 mitochondrial matrix. All these peptides are members of the oxidative

394 phosphorylation complexes (Ojala et al., 1981). Several genetic mutations in nuclear

395 genes coding for mitochondrial proteins or mitochondrial genes that can cause defects

396 in mitochondrial transcripts or mitochondrial proteins leading to mitochondrial 
dysfunction and consequently impaired energy production, have been linked to many

398 inherited diseases (reviewed in (Vafai and Mootha 2012)). Mutations affecting

399 MRPS2 were observed to cause mitochondrial disorder, altered cellular metabolism,

400 developmental delay, and multiple defects in the oxidative phosphorylation system in

401 a study by Gardeitchik and colleagues (Gardeitchik et al., 2018).

402 Given that most cellular functions as well as tight junction maintenance and

403 maintenance of the epithelial barrier are energy-dependent, it could be assumed that

404 mitochondrial dysfunction may play a key role in both the onset and recurrence of

405 IBD. The intestinal mucosa of IBD patients is in a state of energy deficiency,

406 characterized by alterations in the oxidative metabolism of epithelial cells and reduced

407 levels of ATP within the intestine (Roediger 1980; Kameyama et al., 1984; Fukushima

408 and Fiocchi 2004). Several studies have provided evidence of mitochondrial stress and

409 abnormalities within the intestinal epithelium of patients with IBD and mice with

410 experimentally induced colitis (Delpre et al., 1989; Nazli et al., 2004; Rodenburg et

411 al., 2008). The hallmarks of mitochondrial dysfunction, including oxidative stress and

412 impaired ATP production, have been observed in the intestines of patients with IBD

413 (Kruidenier and Verspaget 2002; Pravda 2005; Rezaie, et al., 2007) however, it is yet

414 unclear whether these processes occur as a cause or consequence of the disease.

415 On Chr11, we identified several SNPs within $1 \mathrm{~Kb}$ up- and downstream of genes. Two

416 SNPs with modifier impact within $1 \mathrm{~kb}$ upstream of $I L 3,2$ SNPs downstream of

417 PDLIM and IL13 and one novel SNP downstream of IL4, were identified.

418 Interestingly, all these genes have previously been shown to be associated with human

419 IBD (Jostins et al. 2012). According to our results, the same genes identified as

420 potentially good candidates for IBD in GSDs, further supporting the usefulness of the

421 domestic dog as a natural animal model for human diseases and especially for IBD. 
422 Although disease-associated genetic variations are commonly thought to affect the

423 coding regions of genes, it has been observed that some may alter normal gene

424 expression (Kleinjan and van Heyningen 2005). Thus, it might be possible that the

425 identified SNPs may alter the expression of the genes. It is worth noting that altered

426 expression of interleukins at mRNA and protein levels in human and canine IBD has

427 been reported in several studies.

428 In addition, there is evidence that a conserved noncoding element (CNE) located

429 between IL4 and IL13 controls expression of both genes, as well as IL5. A conserved

430 element was identified by cross-species sequence comparison in the intergenic space

431 between the ILA and IL13 cytokine genes. Targeted deletion in mice revealed it to be

432 a coordinate enhancer for IL4 and IL13, as well as for the more distant IL5 gene. This

433 deletion was also affecting the gene expression in Th2 cells (Loots 2000; Mohrsi et

434 al., 2001).

435 We also assessed whether there is a correlation between variants identified in the case

436 population and response to treatment. The majority of identified SNPs were present in

437 all treatment response groups and therefore we were unable to detect statistically

438 significant differences. Several missense and modifier SNPs were present in either

439 FRD and ARD or SRD and NRS (PTS) group however, these SNPs were present in

440 only one or two cases. Further studies using a bigger sample size are needed to confirm

441 these results, since the lack of association between SNP markers and response to

442 treatment in the present study maybe attributable to small sample size.

443 Canine IBD, similar to the condition in humans, is considered to be a complex

444 multifactorial disorder that seems to occur in genetically susceptible individuals after

445 exposure to one or more environmental triggers. In general, it is believed that a number 
446 of "susceptibility variants" may cause a general predisposition to IBD, and additional

447 genetic variation or environmental factors may influence specific phenotypic

448 characteristics of the individual such as disease site, disease behaviour or response to

449 treatment. In humans, it has been shown that some susceptibility loci are shared

450 between CD and UC, the two major subtypes of IBD, but others are specific to either

451 CD or UC, which perhaps are responsible for diverging disease courses (Satsangi et

452 al., 1997; Ahmad et al., 2001; Abraham and Cho 2009).

453 The results of the present study suggest that the sample size of 28 IBD cases may not

454 have enough power to detect associations of rare alleles with the disease. A larger

455 genotyped population may be necessary due to the complex genetic architecture of the

456 disease and environmental effects. In addition, studying environmental differences in

457 dogs with different responses to treatment may help to identify environmental factors

458 that could affect response to treatment. In humans, a number of associations have been

459 reported between environmental factors such as infections in childhood, diet and

460 smoking, and increased risk of developing IBD and their effect on the efficacy of

461 treatments (Wurzelmann et al., 1994; Chapman-Kiddell et al., 2010; Sandborn et al.,

462 2010; Kiss et al., 2011; Sandborn et al., 2015). Further investigation of potential

463 environmental associations, such as deworming and dietary history, vaccination, as

464 well as previous occurrences of infection in GSDs might therefore help identifying

465 factors affecting treatment response.

466 By performing targeted NGS of the two associated regions identified by GWAS

467 (Peiravan et al., 2018), an attempt was made to identify variants contributing to

468 susceptibility or resistance to canine IBD and then evaluate their correlation with

469 response to treatment. Here, a number of good candidate SNPs with strong association

470 and a potential functional effect were identified. While one or several of these variants 
471 may be the causal variant(s), it is also possible that actual causal variants may have

472 been missed in the targeted re-sequencing process or in the genotyping process for

473 technical reasons. Considering the limited sample size of this study, missing calls in

474 some of the SNPs could affect the significance of the results. Therefore, it may be

475 useful to genotype the cases and controls that have not been called properly at these

476 positions, before performing further investigations. The actual functional variant may

477 also be an indel or CNV which has not been investigated in the current study.

478 A follow-up study in a larger population of IBD cases with different treatment

479 responses is essential to validate results and confirm the variants and genes

480 significantly associated with disease. The SNPs detected by NGS could be further

481 genotyped using a custome made genotyping platform such as Sequenom

482 MassARRAY iPLEX in a larger population of GSDs. In addition, targeted NGS of the

483 other two associated regions on Chr 7 and 13, identified by GWAS will help to identify

484 causal variants and subsequent functional analysis of the causal variants may reveal

485 insights into mechanisms involved in the pathogenesis of canine IBD.

486 The results presented here represent a starting point for further studies of genetic

487 factors associated with canine IBD. Further studies are necessary to conclusively

488 define whether there is a correlation between certain sets of variants, including newly

489 identified variants and previously known variants in Toll-like receptors (TLR)4, 5 and

490 Nucleotide-binding oligomerization domain-containing protein 2 (NOD2) and

491 response to treatment in GSDs with IBD. Identification of variants associated with the

492 disease could potentially lead to the development of a genetic screening test to assist

493 veterinarians with a diagnosis of IBD, and screening for SNPs that are predictive of

494 response to a specific therapy could, potentially maximize treatment efficiency. 
495 Given the heterogeneity of IBD, it is unlikely that any single marker or class of

496 markers could successfully predict response to treatment. However, a combination of

497 several classes of markers, including genetic, serological and inflammatory markers,

498 may have valuable potential to predict the outcome of a treatment. 


\section{Aknoledgement}

500 This work was funded through a BBSRC iCASE studentship (BB/J01236X/1) awarded

501 to K.A. and D.W. with Laboklin GmBH (Bad Kissingen, Germany) as industrial partner

502 as well as the American Kennel Club. We are grateful to owners of GSD who gave

503 permission for their dogs to participate in this study.

\section{Figure Captions}

506 Figure 1. The most highly represented canonical pathways of genes located at the candidate regions.

507 The solid yellow line indicates the significance threshold. The line with squares represents the ratio of 508 the genes represented within each pathway to the total number of genes in the pathway.

509 Figure 2. Cell cycle network and genes involved in the network.

510 Grey filled shapes represent genes included in the list of candidate genes identified in the targeted 511 regions. Solid and dotted lines represent direct and indirect interaction between genes respectively. 
bioRxiv preprint doi: https://doi.org/10.1101/2021.04.20.440584; this version posted April 21, 2021. The copyright holder for this preprint (which was not certified by peer review) is the author/funder, who has granted bioRxiv a license to display the preprint in perpetuity. It is made available under aCC-BY-NC-ND 4.0 International license.

\section{Tables}


bioRxiv preprint doi: https://doi.org/10.1101/2021.04.20.440584; this version posted April 21, 2021. The copyright holder for this preprint (which was not certified by peer review) is the author/funder, who has granted bioRxiv a license to display the preprint in perpetuity. It is made available under aCC-BY-NC-ND 4.0 International license.

513 Table 1. Properties of the capture arrayes designed for isolation of the target regions on 514 Chromosomes 9 and 11.

515

\begin{tabular}{|c|l|l|l|}
\hline Chromosome & \# Markers & Array size & Density \\
\hline $\mathbf{9}$ & 57587 & $1.712 \mathrm{Mb}$ & $2 \mathrm{X}$ tiling \\
\hline $\mathbf{1 1}$ & 30279 & $1.565 \mathrm{Mb}$ & $2 \mathrm{X}$ tiling \\
\hline
\end{tabular}

516 
bioRxiv preprint doi: https://doi.org/10.1101/2021.04.20.440584; this version posted April 21, 2021. The copyright holder for this preprint

(which was not certified by peer review) is the author/funder, who has granted bioRxiv a license to display the preprint in perpetuity. It is made available under aCC-BY-NC-ND 4.0 International license.

517 Table 2. The number of cases in each treatment esponse group.

518 FRD: food responsive disease/diarrhoea, ARD: antibiotic responsive disease/diarrhoea, SRD: steroid

519 responsive disease/diarrhoea, NRS: no response to treatment, PTS: put to sleep (euthanized)

520

521

\begin{tabular}{|l|l|l|}
\hline \multicolumn{2}{|l|}{ Response to treatment } & \# cases \\
\hline Food and antibiotic treated cases & FRD & 9 \\
\cline { 2 - 3 } & ARD & 4 \\
\hline \multirow{2}{*}{ Steroid treated cases } & SRD & 11 \\
\cline { 2 - 3 } & NRS/PTS & 4 \\
\hline
\end{tabular}


bioRxiv preprint doi: https://doi.org/10.1101/2021.04.20.440584; this version posted April 21, 2021. The copyright holder for this preprint

(which was not certified by peer review) is the author/funder, who has granted bioRxiv a license to display the preprint in perpetuity. It is made available under aCC-BY-NC-ND 4.0 International license.

522 Table 3. Number of raw and hard filtered SNPs in the case and control groups in the target

523 region on chromosomes 9 and 11.

524

\begin{tabular}{|c|l|l|l|}
\hline Chromosome & Sample & Raw SNPs & Hard Filtered SNPs \\
\hline $\mathbf{9}$ & Case & 12149 & 10600 \\
\hline \multirow{2}{*11}{} & Control & 12316 & 10908 \\
\hline & Case & 3806 & 2495 \\
\cline { 2 - 4 } & Control & 3884 & 2508 \\
\hline
\end{tabular}


bioRxiv preprint doi: https://doi.org/10.1101/2021.04.20.440584; this version posted April 21, 2021. The copyright holder for this preprint

(which was not certified by peer review) is the author/funder, who has granted bioRxiv a license to display the preprint in perpetuity. It is made available under aCC-BY-NC-ND 4.0 International license.

527 Table 4. Number of SNPs in each group on Chr 9 and 11.

528 Case only: SNPs that were only present in case population, Ctrl only: SNPs present only in control

529 population, Overlapped with different Alt in Case/ or Ctrl: SNPs that were present in both case and

530 control population but showing different alternate allele in case and control, Overlapped with same Alt

531 in Case and Ctrl: SNPs that were present in both case and control population and also showing same

532 alternate allele in both case and control. Chr: chromosome, Alt: alternate, Ctrl: control.

\begin{tabular}{|l|l|l|l|l|l|}
\hline Chromosome & $\begin{array}{l}\text { Case } \\
\text { only }\end{array}$ & $\begin{array}{l}\text { Control } \\
\text { only }\end{array}$ & $\begin{array}{l}\text { Overlapped } \\
\text { with } \\
\text { different Alt } \\
\text { in Case }\end{array}$ & $\begin{array}{l}\text { Overlapped } \\
\text { with } \\
\text { different Alt } \\
\text { in Ctrl }\end{array}$ & $\begin{array}{l}\text { Overlapped } \\
\text { with same Alt } \\
\text { in Case and } \\
\text { Ctrl }\end{array}$ \\
\hline $\mathbf{9}$ & 985 & 1293 & 14 & 14 & 9601 \\
\hline $\mathbf{1 1}$ & 721 & 734 & 6 & 6 & 1768 \\
\hline
\end{tabular}




\begin{tabular}{|c|c|c|c|c|c|c|c|c|c|c|c|c|c|c|}
\hline \multirow[b]{2}{*}{ Variation } & \multirow[b]{2}{*}{ Location } & \multirow[b]{2}{*}{ Allele } & \multirow[b]{2}{*}{ Consequence } & \multirow[b]{2}{*}{ Impact } & \multirow[b]{2}{*}{ Symbol } & \multicolumn{5}{|c|}{ Case } & \multicolumn{4}{|c|}{ Control } \\
\hline & & & & & & $\begin{array}{l}\text { No } \\
\text { call }\end{array}$ & $\begin{array}{l}\text { Hom } \\
\text { Ref }\end{array}$ & Variant & Het & Homo & $\begin{array}{l}\text { No } \\
\text { call }\end{array}$ & $\begin{array}{l}\text { Hom } \\
\text { Ref }\end{array}$ & Variant & $\mathrm{P}$ value \\
\hline . & 9:50769631 & G*/A & Upstream & MODIFIER & COL5A1 & 3 & 14 & 11 & 10 & 1 & 1 & 19 & 0 & 0.0009 \\
\hline . & 9:51215957 & $\mathrm{T}^{*} / \mathrm{C}$ & downstream & MODIFIER & MRPS2 & 0 & 20 & 8 & 8 & 0 & 3 & 17 & 0 & 0.017 \\
\hline rs24555947 & 9:51227287 & $\mathrm{C}^{*} / \mathrm{G}$ & downstream & MODIFIER & EEF1A1 & 0 & 12 & 16 & 16 & 0 & 0 & 20 & 0 & $2 \mathrm{E}-05$ \\
\hline . & 9:51227325 & $\mathrm{G}^{*} / \mathrm{A}$ & downstream & MODIFIER & EEF1A1 & 3 & 6 & 19 & 19 & 0 & 2 & 18 & 0 & $6.68 \mathrm{E}-07$ \\
\hline . & 9:51227411 & $\mathrm{T}^{*} / \mathrm{A}$ & downstream & MODIFIER & EEF1A1 & 0 & 2 & 26 & 26 & 0 & 0 & 20 & 0 & $1.38 \mathrm{E}-11$ \\
\hline . & 9:51228186 & $\mathrm{C}^{*} / \mathrm{T}$ & stop_gained & HIGH & EEF1A1 & 0 & 2 & 26 & 26 & 0 & 0 & 20 & 0 & $1.38 \mathrm{E}-11$ \\
\hline rs24570325 & $9: 51228828$ & $\mathrm{G}^{*} / \mathrm{T}$ & Upstream & MODIFIER & EEF1A1 & 9 & 12 & 7 & 4 & 3 & 0 & 20 & 0 & 0.003 \\
\hline rs24570326 & 9:51228829 & $\mathrm{C}^{*} / \mathrm{T}$ & Upstream & MODIFIER & EEF1A1 & 9 & 12 & 7 & 4 & 3 & 0 & 20 & 0 & 0.003 \\
\hline . & 9:51255622 & $\mathrm{T}^{*} / \mathrm{C}$ & Upstream & MODIFIER & Novel gene & 0 & 17 & 11 & 9 & 2 & 0 & 20 & 0 & 0.001 \\
\hline . & 9:51404724 & $\mathrm{C}^{*} / \mathrm{T}$ & Missense & MODERATE & MDH2 & 7 & 3 & 18 & 18 & 0 & 1 & 19 & 0 & 7.52E-09 \\
\hline . & 9:51405189 & $\mathrm{T}^{*} / \mathrm{C}$ & downstream & MODIFIER & MDH2 & 4 & 1 & 23 & 23 & 0 & 1 & 19 & 0 & $2.50 \mathrm{E}-11$ \\
\hline rs852815996 & 9:51994360 & $\mathrm{T} * / \mathrm{C}$ & downstream & MODIFIER & NTNG2 & 8 & 8 & 12 & 2 & 10 & 3 & 17 & 0 & 0.00007 \\
\hline
\end{tabular}


542 Hom Ref: sites with reference allele (AA), Het: Heterozygous (AB), Homo: Homozygous (BB), P value: Fisher exact probability test two tailed p value.

543 IL3: Interleukin 3, P4HA2: Prolyl 4-hydroxylase subunit alpha-2, PDLIM4: PDZ and LIM domain protein 4, IL13: Interleukine 13. *Genes already found to be associated with 544 human IBD.

\begin{tabular}{|c|c|c|c|c|c|c|c|c|c|c|c|c|c|c|}
\hline \multirow[t]{2}{*}{ Variation } & \multirow[b]{2}{*}{ Location } & \multirow[b]{2}{*}{ Allele } & \multirow[b]{2}{*}{ Consequence } & \multirow[b]{2}{*}{ Impact } & \multirow[b]{2}{*}{ Symbol } & \multicolumn{5}{|c|}{ Case } & \multicolumn{3}{|c|}{ Control } & \multirow[b]{2}{*}{$\mathrm{P}$ value } \\
\hline & & & & & & $\begin{array}{l}\text { No } \\
\text { call }\end{array}$ & $\begin{array}{l}\text { Hom } \\
\text { Ref }\end{array}$ & Variant & Het & Homo & $\begin{array}{l}\text { No } \\
\text { call }\end{array}$ & $\begin{array}{l}\text { Hom } \\
\text { Ref }\end{array}$ & Variant & \\
\hline rs851236833 & $11: 20329483$ & C*/A & upstream & Modifier & IL $3 *$ & 3 & 13 & 12 & 10 & 2 & 3 & 17 & 0 & 0.001 \\
\hline rs853055449 & 11:20329514 & $\mathrm{A} * / \mathrm{C}$ & upstream & Modifier & IL $3 *$ & 3 & 13 & 12 & 10 & 2 & 2 & 18 & 0 & 0.0003 \\
\hline rs852084335 & $11: 20549348$ & $\mathrm{~T}^{*} / \mathrm{C}$ & upstream & Modifier & P4HA2 & 2 & 14 & 12 & 11 & 1 & 2 & 18 & 0 & 0.001 \\
\hline rs850644440 & $11: 20587679$ & C*/A & downstream & Modifier & PDLIM4* & 9 & 10 & 9 & 6 & 3 & 3 & 17 & 0 & 0.001 \\
\hline rs22191752 & 11:20962569 & $\mathrm{T}^{*} / \mathrm{C}$ & downstream & Modifier & IL13* & 2 & 12 & 14 & 6 & 8 & 3 & 17 & 0 & 0.0001 \\
\hline
\end{tabular}




\begin{tabular}{|c|c|c|c|c|c|c|c|c|c|c|}
\hline \multirow[t]{2}{*}{ Variation } & & \multicolumn{4}{|c|}{ Alleles } & \multicolumn{5}{|c|}{ Genotype } \\
\hline & Location & Allele & & Maf & No call & Hom & Variant & Het & Homo & Impact/Symbol \\
\hline \multirow[t]{2}{*}{ rs22147008 } & 11:20960082 & $A * / G$ & Case & 0.054 & 0 & 25 & 3 & 3 & 0 & \multirow{2}{*}{$\begin{array}{l}\text { Moderate, } \\
\text { deleterious }(0.04) / I L 13\end{array}$} \\
\hline & & & CTRL & 0.074 & 0 & 17 & 3 & 3 & 0 & \\
\hline
\end{tabular}




\section{References}

Abraham, Clara, and Judy H. Cho. 2009. "Inflammatory Bowel Disease." New England Journal of Medicine $361 \quad$ (21): 2066-78. https://doi.org/10.1056/NEJMra0804647.

Ahmad, T, J Satsangi, D McGovern, M Bunce, and D P Jewell. 2001. "Review Article: The Genetics of Inflammatory Bowel Disease." Alimentary Pharmacology \& Therapeutics 15 (6): 731-48.

Albert, E. Jergens. 1999. "Inflammatory Bowel Disease: Current Perspectives." Veterinary Clinics of North America: Small Animal Practice 29 (2): 501-21. https://doi.org/10.1016/S0195-5616(99)50032-6.

Allenspach, K., S. Rüfenacht, S. Sauter, A. Gröne, J. Steffan, G. Strehlau, and F. Gaschen. 2006. "Pharmacokinetics and Clinical Efficacy of Cyclosporine Treatment of Dogs with Steroid-Refractory Inflammatory Bowel Disease." Journal of Veterinary Internal Medicine $20 \quad$ (2): 239-44. https://doi.org/10.1111/j.1939-1676.2006.tb02852.x.

Allenspach, K, B Wieland, A Gröne, and F Gaschen. 2007. "Chronic Enteropathies in Dogs: Evaluation of Risk Factors for Negative Outcome.” Journal of Veterinary $\begin{array}{llll}\text { Internal } & \text { Medicine } & 21 & \text { 700-708. }\end{array}$ http://www.ncbi.nlm.nih.gov/pubmed/17708389.

Bolger, Anthony M., Marc Lohse, and Bjoern Usadel. 2014. "Trimmomatic: A Flexible Trimmer for Illumina Sequence Data.” Bioinformatics 30 (15): 211420. https://doi.org/10.1093/bioinformatics/btu170.

Cacquevel, Mathias, Nathalie Lebeurrier, Simon Cheenne, and Denis Vivien. 2004. 
"Cytokines in Neuroinflammation and Alzheimers Disease." Current Drug Targets 5 (6): 529-34. https://doi.org/10.2174/1389450043345308.

Chapman-Kiddell, Christine A, Peter S W Davies, Lynda Gillen, and Graham L Radford-Smith. 2010. "Role of Diet in the Development of Inflammatory Bowel Disease." Inflammatory Bowel Diseases $16 \quad(1)$ 137-51. https://doi.org/10.1002/ibd.20968.

Delpre, G, I Avidor, R Steinherz, U Kadish, and M Ben-Bassat. 1989. “Ultrastructural Abnormalities in Endoscopically and Histologically Normal and Involved Colon in Ulcerative Colitis.” The American Journal of Gastroenterology 84 (9): 103846. http://www.ncbi.nlm.nih.gov/pubmed/2773897.

Fukushima, Kouhei, and Claudio Fiocchi. 2004. "Paradoxical Decrease of Mitochondrial DNA Deletions in Epithelial Cells of Active Ulcerative Colitis Patients." American Journal of Physiology-Gastrointestinal and Liver Physiology 286 (5): G804-13. https://doi.org/10.1152/ajpgi.00398.2003.

Gardeitchik, Thatjana, Miski Mohamed, Benedetta Ruzzenente, Daniela Karall, Sergio Guerrero-Castillo, Daisy Dalloyaux, Mariël van den Brand, et al. 2018. "Bi-Allelic Mutations in the Mitochondrial Ribosomal Protein MRPS2 Cause Sensorineural Hearing Loss, Hypoglycemia, and Multiple OXPHOS Complex Deficiencies." The American Journal of Human Genetics 102 (4): 685-95. https://doi.org/10.1016/j.ajhg.2018.02.012.

German, A.J., M.J. Day, C.G. Ruaux, J.M. Steiner, D.A. Williams, and E.J. Hall. 2003. "Comparison of Direct and Indirect Tests for Small Intestinal Bacterial Overgrowth and Antibiotic-Responsive Diarrhea in Dogs." Journal of Veterinary Internal Medicine $17 \quad$ (1): $\quad 33-43 . \quad$ https://doi.org/10.1111/j.1939- 
1676.2003.tb01321.x.

Guilford WG. 1996. "Idiopathic Inflammatory Bowel Diseases.” Strombecks Small Animal Gastroenterology, 451-86.

Hendrickson, B. A., R. Gokhale, and J. H. Cho. 2002. "Clinical Aspects and Pathophysiology of Inflammatory Bowel Disease.” Clinical Microbiology Reviews 15 (1): 79-94. https://doi.org/10.1128/CMR.15.1.79-94.2002.

Hsieh, Sen-Yung, Tsung-Chieh Shih, Chien-Yuh Yeh, Chun-Jung Lin, Yun-Ying Chou, and Ying-Shiung Lee. 2006. "Comparative Proteomic Studies on the Pathogenesis of Human Ulcerative Colitis.” Proteomics 6 (19): 5322-31. https://doi.org/10.1002/pmic.200500541.

Ito, Hiroaki. 2004. "Novel Therapy for Crohn’s Disease Targeting IL-6 Signalling.” Expert Opinion on Therapeutic Targets 8 (4): 287-94. https://doi.org/10.1517/14728222.8.4.287.

Jostins, Luke, Stephan Ripke, Rinse K Weersma, Richard H Duerr, Dermot P McGovern, Ken Y Hui, James C Lee, et al. 2012. "Host-Microbe Interactions Have Shaped the Genetic Architecture of Inflammatory Bowel Disease.” Nature 491 (7422): 119-24. https://doi.org/10.1038/nature11582.

KAMEYAMA, JIN-ICHI, HIDEO NARUI, MASARU INUI, and TOSHIO SATO. 1984. "Energy Level in Large Intestinal Mucosa in Patients with Ulcerative Colitis." The Tohoku Journal of Experimental Medicine 143 (2): 253-54. https://doi.org/10.1620/tjem.143.253.

Kapp, Lee D, and Jon R Lorsch. 2004. "The Molecular Mechanics of Eukaryotic Translation." Annual Review of Biochemistry 73: 657-704. 
https://doi.org/10.1146/annurev.biochem.73.030403.080419.

Karlsson, Elinor K, Izabella Baranowska, Claire M Wade, Nicolette H C Salmon Hillbertz, Michael C Zody, Nathan Anderson, Tara M Biagi, et al. 2007. "Efficient Mapping of Mendelian Traits in Dogs through Genome-Wide Association." $\quad$ Nature $\quad$ Genetics $\quad 39 \quad$ (11): 1321-28. https://doi.org/10.1038/ng.2007.10.

Kathrani, A., A. House, B. Catchpole, A. Murphy, D. Werling, and K. Allenspach. 2011. "Breed-Independent Toll-like Receptor 5 Polymorphisms Show Association with Canine Inflammatory Bowel Disease.” Tissue Antigens 78 (2): 94-101. https://doi.org/10.1111/j.1399-0039.2011.01707.x.

Kathrani, A., H. Lee, C. White, B. Catchpole, A. Murphy, A. German, D. Werling, and K. Allenspach. 2014. "Association between Nucleotide Oligomerisation Domain Two (Nod2) Gene Polymorphisms and Canine Inflammatory Bowel Disease." Veterinary Immunology and Immunopathology 161 (1-2): 32-41. https://doi.org/10.1016/j.vetimm.2014.06.003.

Kathrani, A., D. Werling, and K. Allenspach. 2011. "Canine Breeds at High Risk of Developing Inflammatory Bowel Disease in the South-Eastern UK.”Veterinary Record 169 (24): 635-635. https://doi.org/10.1136/vr.d5380.

Kathrani, Aarti, Arthur House, Brian Catchpole, Angela Murphy, Alex German, Dirk Werling, and Karin Allenspach. 2010. "Polymorphisms in the Tlr4 and Tlr5 Gene Are Significantly Associated with Inflammatory Bowel Disease in German $\begin{array}{lllll}\text { Shepherd Dogs.” } & P L o S & O N E & 5 & \text { (12): }\end{array}$ https://doi.org/10.1371/journal.pone.0015740.

Kimmel, Susan E., Lori S. Waddell, and Kathryn E. Michel. 2000. "Hypomagnesemia 
and Hypocalcemia Associated with Protein-Losing Enteropathy in Yorkshire Terriers: Five Cases (1992-1998)." Journal of the American Veterinary Medical Association 217 (5): 703-6. https://doi.org/10.2460/javma.2000.217.703.

Kiss, L S, T Szamosi, T Molnar, P Miheller, L Lakatos, A Vincze, K Palatka, et al. 2011. "Early Clinical Remission and Normalisation of CRP Are the Strongest Predictors of Efficacy, Mucosal Healing and Dose Escalation during the First Year of Adalimumab Therapy in Crohn's Disease." Alimentary Pharmacology \& Therapeutics 34 (8): 911-22. https://doi.org/10.1111/j.1365-2036.2011.04827.x.

Kleinjan, Dirk A., and Veronica van Heyningen. 2005. "Long-Range Control of Gene Expression: Emerging Mechanisms and Disruption in Disease." The American Journal of Human Genetics 76 (1): 8-32. https://doi.org/10.1086/426833.

Kobayashi, Y, and \& S Yonehara. 2009. "Novel Cell Death by Downregulation of EEF1A1 Expression in Tetraploids." Cell Death and Differentiation 16: 139150.

Kruidenier, L, and H W Verspaget. 2002. "Review Article: Oxidative Stress as a Pathogenic Factor in Inflammatory Bowel Disease--Radicals or Ridiculous?" Alimentary Pharmacology \& Therapeutics 16 (12): 1997-2015. http://www.ncbi.nlm.nih.gov/pubmed/12452933.

Lequarré, Anne-Sophie, Leif Andersson, Catherine André, Merete Fredholm, Christophe Hitte, Tosso Leeb, Hannes Lohi, Kerstin Lindblad-Toh, and Michel Georges. 2011. "LUPA: A European Initiative Taking Advantage of the Canine Genome Architecture for Unravelling Complex Disorders in Both Human and Dogs." The Veterinary Journal $189 \quad$ (2): $155-59$. https://doi.org/10.1016/j.tvj1.2011.06.013. 
Li, H., B. Handsaker, A. Wysoker, T. Fennell, J. Ruan, N. Homer, G. Marth, G. Abecasis, and R. Durbin. 2009. "The Sequence Alignment/Map Format and SAMtools." $\quad$ Bioinformatics $\quad 25 \quad$ (16): $2078-79$. https://doi.org/10.1093/bioinformatics/btp352.

Li, Heng, and Richard Durbin. 2009. "Fast and Accurate Short Read Alignment with Burrows-Wheeler Transform.” Bioinformatics 25 (14): 1754-60. https://doi.org/10.1093/bioinformatics/btp324.

Lindblad-Toh, Kerstin, Claire M. Wade, Tarjei S. Mikkelsen, Elinor K. Karlsson, David B. Jaffe, Michael Kamal, Michele Clamp, et al. 2005. “Genome Sequence, Comparative Analysis and Haplotype Structure of the Domestic Dog." Nature 438 (7069): 803-19. https://doi.org/10.1038/nature04338.

Loots, G. G. 2000. "Identification of a Coordinate Regulator of Interleukins 4,\&amp;Nbsp;13,\&amp;Nbsp;and 5\&amp;Nbsp;by Cross-Species Sequence Comparisons." $\quad$ Science $\quad 288 \quad$ (5463): $\quad$ 136-40. https://doi.org/10.1126/science.288.5463.136.

Luckschander, Nicole, Karin Allenspach, Jean Hall, Frank Seibold, Andrea Gröne, Marcus G. Doherr, and Frédéric Gaschen. 2006. "Perinuclear Antineutrophilic Cytoplasmic Antibody and Response to Treatment in Diarrheic Dogs with Food Responsive Disease or Inflammatory Bowel Disease.” Journal of Veterinary Internal Medicine $20 \quad$ (2): 221-27. https://doi.org/10.1892/08916640(2006)20[221:PACAAR]2.0.CO;2.

M Mohrs, CM Blankespoor, ZE Wang, GG Loots, V Afzal, H Hadeiba, K Shinkai, EM Rubin, RM Locksley. 2001. "Deletion of a Coordinate Regulator of Type 2 Cytokine Expression in Mice.” Nature Immunology 2: 842-47. 
Mandigers, P.J.J., V. Biourge, T.S.G.A.M. Van Den Ingh, N. Ankringa, and A.J. German. 2010. “A Randomized, Open-Label, Positively-Controlled Field Trial of a Hydrolyzed Protein Diet in Dogs with Chronic Small Bowel Enteropathy.” Journal of Veterinary Internal Medicine 24 (6): 1350-57. https://doi.org/10.1111/j.1939-1676.2010.0632.x.

Murphy, Stephen F., John H. Kwon, and David L. Boone. 2012. "Novel Players in Inflammatory Bowel Disease Pathogenesis.” Current Gastroenterology Reports 14 (2): 146-152.

Nazli, Aisha, Ping-Chang Yang, Jennifer Jury, Kathryn Howe, James L. Watson, Johan D. Söderholm, Philip M. Sherman, Mary H. Perdue, and Derek M. McKay. 2004. "Epithelia Under Metabolic Stress Perceive Commensal Bacteria as a Threat." The American Journal of Pathology 164 (3): 947-57. https://doi.org/10.1016/S0002-9440(10)63182-3.

Negrutskii, Boris, Dmytro Vlasenko, and Anna El'skaya. 2012. "From Global Phosphoproteomics to Individual Proteins: The Case of Translation Elongation Factor EEF1A." Expert Review of Proteomics 9 (1): 71-83. https://doi.org/10.1586/epr.11.71.

Nishimoto, Norihiro. 2006. "Interleukin-6 in Rheumatoid Arthritis." Current Opinion $\begin{array}{llll}\text { in } & \text { Rheumatology } & 18 & \text { (3): }\end{array}$ https://doi.org/10.1097/01.bor.0000218949.19860.d1.

Ojala, D, J Montoya, and G Attardi. 1981. "TRNA Punctuation Model of RNA Processing in Human Mitochondria." Nature 290 (5806): 470-74. https://doi.org/7219536.

Peiravan, Atiyeh, Karin Allenspach, Alisdair M. Boag, Francesca Soutter, Angela 
Holder, Brian Catchpole, Lorna J. Kennedy, Dirk Werling, and Fabio Procoli. 2016. "Single Nucleotide Polymorphisms in Major Histocompatibility Class II Haplotypes Are Associated with Potential Resistance to Inflammatory Bowel Disease in German Shepherd Dogs." Veterinary Immunology and Immunopathology

https://doi.org/10.1016/j.vetimm.2016.10.012.

Peiravan, Atiyeh, Francesca Bertolini, Max F. Rothschild, Kenneth W. Simpson, Albert E. Jergens, Karin Allenspach, and Dirk Werling. 2018. "Genome-Wide Association Studies of Inflammatory Bowel Disease in German Shepherd Dogs.” Edited by John Green. PLOS ONE $13 \quad$ (7): 0200685. https://doi.org/10.1371/journal.pone.0200685.

Pravda, Jay. 2005. "Radical Induction Theory of Ulcerative Colitis.” World Journal of $\quad$ Gastroenterology $\quad 11 \quad$ (16): $2371-84$. http://www.ncbi.nlm.nih.gov/pubmed/15832404.

Rezaie, Ali, Robyn D Parker, and Mohammad Abdollahi. 2007. "Oxidative Stress and Pathogenesis of Inflammatory Bowel Disease: An Epiphenomenon or the Cause?" Digestive Diseases and Sciences 52 (9): 2015-21. https://doi.org/10.1007/s10620-006-9622-2.

Roberts, Rebecca L, and Murray L Barclay. 2012. "Current Relevance of Pharmacogenetics in Immunomodulation Treatment for Crohn's Disease." Journal of Gastroenterology and Hepatology 27 (10): 1546-54. https://doi.org/10.1111/j.1440-1746.2012.07220.x.

Rodenburg, Wendy, Jaap Keijer, Evelien Kramer, Carolien Vink, Roelof van der Meer, and Ingeborg MJ Bovee-Oudenhoven. 2008. "Impaired Barrier Function 
by Dietary Fructo-Oligosaccharides (FOS) in Rats Is Accompanied by Increased

Colonic Mitochondrial Gene Expression." BMC Genomics 9 (1): 144. https://doi.org/10.1186/1471-2164-9-144.

Roediger, W.E.W. 1980. “THE COLONIC EPITHELIUM IN ULCERATIVE COLITIS: AN ENERGY-DEFICIENCY DISEASE?” The Lancet 316 (8197): 712-15. https://doi.org/10.1016/S0140-6736(80)91934-0.

Sandborn, W. J., G. Y. Melmed, D. P. B. McGovern, E. V. Loftus, J. M. Choi, J. H. Cho, B. Abraham, et al. 2015. "Clinical and Demographic Characteristics Predictive of Treatment Outcomes for Certolizumab Pegol in Moderate to Severe Crohn's Disease: Analyses from the 7-Year PRECiSE 3 Study." Alimentary Pharmacology \& Therapeutics $42 \quad$ (3): $\quad 330-42$. https://doi.org/10.1111/apt.13251.

Sandborn, William J. 2004. "Pharmacogenomics and IBD." Inflammatory Bowel Diseases 10 (February): S35-37. https://doi.org/10.1097/00054725-20040200100008.

Sandborn, William J., Maria T. Abreu, Geert R. D’Haens, Jean-Frederic Colombel, Severine Vermeire, Krassimir Mitchev, Etienne Ernault, et al. 2010. “S1030 Predictors of Response and Remission to Certolizumab Pegol in Patients With Crohn's Disease: Data From the WELCOME Study.” Gastroenterology 138 (5): S-164. https://doi.org/10.1016/S0016-5085(10)60750-0.

Satsangi, J, D P Jewell, and J I Bell. 1997. "The Genetics of Inflammatory Bowel Disease." Gut 40 (5): 572-74. https://doi.org/10.1136/gut.40.5.572.

Schulz, Ingo, Claudia Engel, André J. Niestroj, Astrid Kehlen, Jens-Ulrich Rahfeld, Martin Kleinschmidt, Karola Lehmann, Steffen Roßner, and Hans-Ulrich 
Demuth. 2014. “A Non-Canonical Function of Eukaryotic Elongation Factor 1A1: Regulation of Interleukin-6 Expression.” Biochimica et Biophysica Acta (BBA) - Molecular Cell Research $1843 \quad$ (5): 965-75. https://doi.org/10.1016/J.BBAMCR.2014.01.022.

Simpson, Kenneth W, Belgin Dogan, Mark Rishniw, Richard E Goldstein, Suzanne Klaessig, Patrick L McDonough, Alex J German, et al. 2006. “Adherent and Invasive Escherichia Coli Is Associated with Granulomatous Colitis in Boxer Dogs." Infection and Immunity 74 (8): 4778-92. https://doi.org/10.1128/IAI.00067-06.

Sutter, N. B. 2004. "Extensive and Breed-Specific Linkage Disequilibrium in Canis Familiaris." Genome Research $14 \quad$ (12): 2388-96. https://doi.org/10.1101/gr.3147604.

Tengvall, Katarina, Marcin Kierczak, Kerstin Bergvall, Mia Olsson, Marcel Frankowiack, Fabiana H.G. Farias, Gerli Pielberg, et al. 2013. "Genome-Wide Analysis in German Shepherd Dogs Reveals Association of a Locus on CFA 27 with Atopic Dermatitis." PLoS Genetics 9 (5): 1-12. https://doi.org/10.1371/journal.pgen.1003475.

Vafai, Scott B., and \& Vamsi K. Mootha. 2012. "Mitochondrial Disorders as Windows into an Ancient Organelle." Naure 491: 374-383.

Westermarck, Elias, Teresa Skrzypczak, Jaana Harmoinen, Jörg M. Steiner, Craig G. Ruaux, David A. Williams, Erkki Eerola, Pernilla Sundbäck, and Minna Rinkinen. 2005. "Tylosin-Responsive Chronic Diarrhea in Dogs.” Journal of Veterinary Internal Medicine 19 (2): 177-86. https://doi.org/10.1111/j.19391676.2005.tb02679.x. 
Wilbe, Maria, Päivi Jokinen, Katarina Truvé, Eija H Seppala, Elinor K Karlsson, Tara

Biagi, Angela Hughes, et al. 2010. "Genome-Wide Association Mapping Identifies Multiple Loci for a Canine SLE-Related Disease Complex.” Nature Genetics 42 (3): 250-54. https://doi.org/10.1038/ng.525.

Wood, Shona Hiedi, Xiayi Ke, Tim Nuttall, Neil McEwan, William E. Ollier, and Stuart D. Carter. 2009. “Genome-Wide Association Analysis of Canine Atopic Dermatitis and Identification of Disease Related SNPs.” Immunogenetics 61 (1112): 765-72. https://doi.org/10.1007/s00251-009-0402-y.

Wurzelmann, John I., Cynthia M. Lyles, and Robert S. Sandler. 1994. "Childhood Infections and the Risk of Inflammatory Bowel Disease." Digestive Diseases and Sciences 39 (3): 555-60. https://doi.org/10.1007/BF02088342.

Ziegler, Andreas, Kristel Van Steen, and Stefan Wellek. 2011. "Investigating HardyWeinberg Equilibrium in Case-control or Cohort Studies or Meta-Analysis." Breast Cancer Research and Treatment 128 (1): 197-201. https://doi.org/10.1007/s10549-010-1295-Z. 


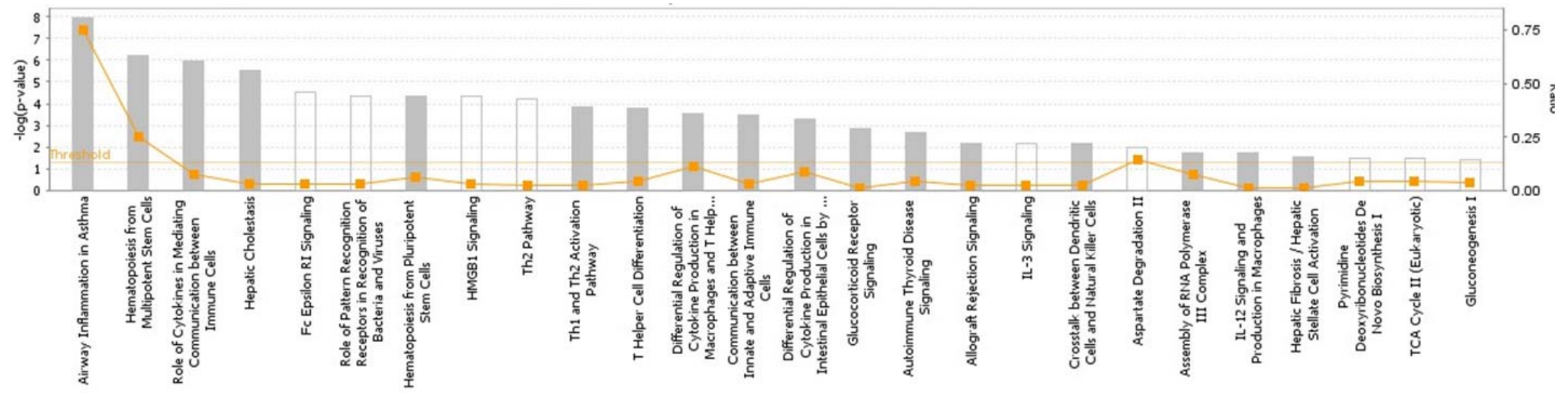

Figure 1 . The most highly represented canonical pathways of genes located at the candidate regions.

The solid yellow line indicates the significance threshold. The line with squares represents the ratio of the genes represented within each pathway to the total number of genes in the pathway. 


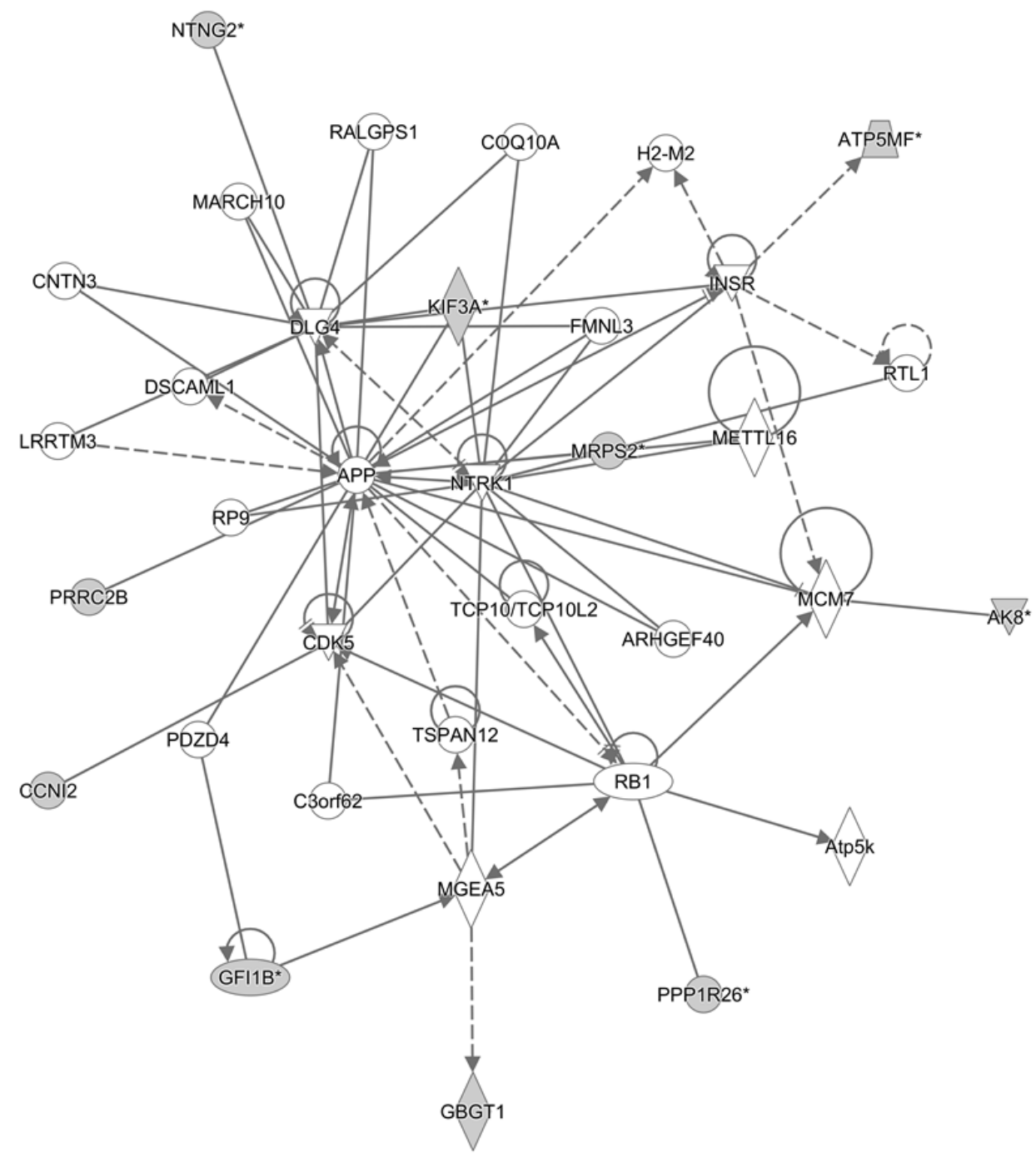

Figure 1. Cell cycle network and genes involved in the network.

Grey filled shapes represent genes included in the list of candidate genes identified in the targeted regions. Solid and dotted lines represent direct and indirect interaction between genes respectively. 